Theresa Thunig, Kai Nagel

\title{
Effects of user adaption on traffic- responsive signal control in agent-based transport simulations
}

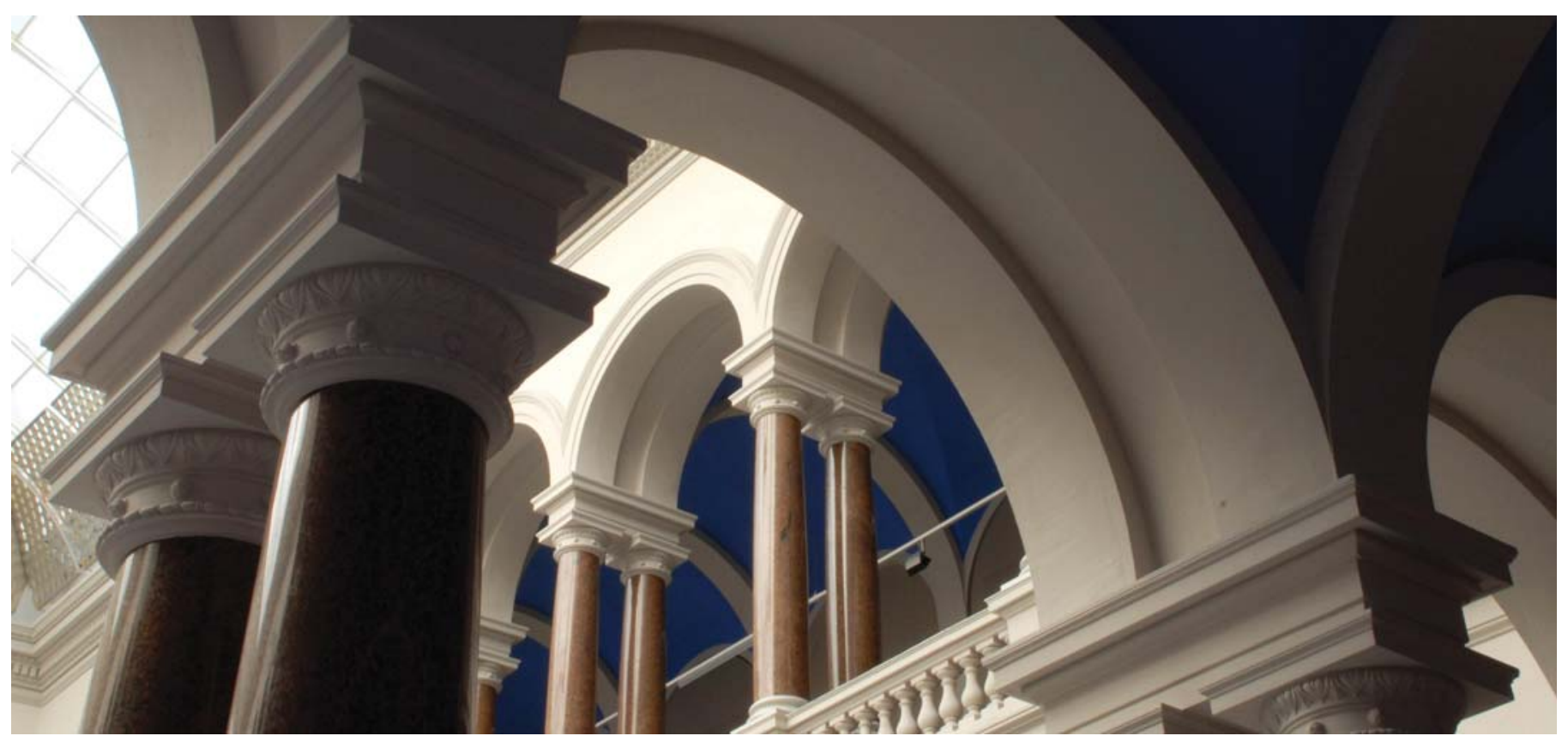

Thunig, T., \& Nagel, K. (2019). Effects of user adaption on traffic-responsive signal control in agent-based transport simulations. 2019 6th International Conference on Models and Technologies for Intelligent Transportation Systems (MT-ITS). https://doi.org/10.1109/mtits.2019.8883373 


\section{Effects of user adaption on traffic-responsive signal control in agent-based transport simulations}

\author{
$1^{\text {st }}$ Theresa Thunig \\ Transport Systems Planning and Transport Telematics \\ Technische Universitt Berlin \\ Berlin, Germany \\ thunig@vsp.tu-berlin.de
}

\author{
$2^{\text {nd }}$ Kai Nagel \\ Transport Systems Planning and Transport Telematics \\ Technische Universitt Berlin \\ Berlin, Germany \\ nagel@vsp.tu-berlin.de
}

\begin{abstract}
All traffic-responsive approaches have in common that they directly influence waiting times of travelers (users) at intersections and, thereby, influence user reaction, e.g. route choice. On the other hand, users route choice directly influences sensor data and, thereby, the signal settings controlled by trafficresponsive signals. Thus, the interference of route choice and traffic-responsive signals constitutes a combined problem. This work focuses on a detailed simulation-based analysis of the effects of route choice on the performance of different traffic-responsive signal algorithms implemented in an inner-city area of a realworld scenario. It Is found that the effects of induced traffic matter a lot, especially for the inner-city area: A significantly higher number of agents travel through the inner city, increasing travel time, delay and noise levels in this area (in comparison to the case without user adoption), whereas overall traveled distances decrease, i.e. more direct routes are used and by-pass routes around the city become less congested. Furthermore, the effects of different levels of saturation on the interaction of route choice and signal control are analyzed.

Index Terms-induced traffic, user adaption, route choice, traffic-responsive signals, agent-based transport simulation, realworld application
\end{abstract}

\section{INTRODUCTION}

Traffic-responsive signals are becoming more and more popular, especially in cities where their ability to directly react to fluctuating demand is particularly useful. However, not only traffic signals react to travelers' behavior also travelers react to signal control, because they influence travel and waiting times. This has to be taken into account when designing and modeling traffic control. In models that only consider current traffic demand and do not account for user reaction, traffic control policies cannot be evaluated correctly: high improvements in travel times might be observed due to better signal control, whereas this effect vanishes in reality, because of induced traffic which, in turn, balances travel times by increased delays. As a consequence, improvements in inner city traffic control are likely to attract more traffic through the inner city, more congestion, and increased emissions, such as noise or NOx.

The authors thank DFG (Deutsche Forschungsgemeinschaft) for funding the project Optimization and network wide analysis of traffic signal control.

978-1-5386-9484-8/19/\$31.00 @2019 IEEE
Who follows whom in the combined problem of trafficsignal and user reaction? With fixed-time signals, users follow signals, because signals are not able to react to changes in demand. In transport modeling, it is usually assumed that travelers act selfishly and choose the option (route, departure time, transport mode etc.) by which they can unilaterally maximize their utility (depending on e.g. travel time and cost). The situation which satisfies this criterion is called user equilibrium. The optimization of fixed-time signals under the assumption of selfish users is not easy. A MIP-based approach has been developed by Strehler and Köhler [1], but is not easy to apply for complex real-world applications [2]. A simulationbased optimization approach has been developed by Osorio and Bierlaire [3].

With traffic-responsive signals, the situation becomes even more complex from an optimization perspective: Signals now follow users, and (because signals directly affect travel times) also users follow signals. I.e. it becomes a combined problem of signal and user reaction. A highly idealized model of this complex combined process has been studied e.g. by Smith and Van Vuren [4] and Zuylen, Taale and Stackelberg [5]. Alternatively, the combined process of traffic-responsive signal and user reaction can be observed and analyzed in transport simulations. With the transport simulation MATSim, for example, it is possible to model various types of user reactions (such as route choice, departure time choice, transport mode choice etc.). Other simulation models, such as SUMO or VISSIM, do not have this possibility in their standard implementation, but provide extensions for route choice modules.

This paper analyzes the combined process of trafficresponsive signal control and user reaction in terms of route choice with the agent-based transport simulation MATSim. A real-world scenario of a city and its surrounding area is used. Two different kinds of traffic-responsive signal algorithms are implemented at the signalized intersections of the inner city. Travel times without and with user reaction are compared to the base case (with fixed-time signal plans). It is found that re-routed traffic (called induced traffic here) has a high impact on the performance of traffic-responsive signal control: The number of trips through the inner city increase, traveled distances decrease (i.e. users choose more direct routes) and travel times and delay increase in the inner-city area. With 
that, also noise levels in the inner city increase, whereas by-pass routes around the inner city become less congested. Furthermore, the paper analyzes the effect of different levels of saturation on the interaction of route choice and signal control. The paper is organized as follows: The transport simulation and the traffic-responsive signal control algorithms are described in Sec. II. Sec. III-A presents the real-world scenario. In Sec. III-B the effects of induced traffic on the performance of traffic-responsive signals are presented and discussed in detail, whereas Sec. III-C analyzes the effect of different levels of saturation on the results. Sec. IV summarizes the findings and highlights possible next steps.

\section{PRELIMINARIES}

\section{A. The multi-agent transport simulation MATSim}

In MATSim [6] traffic is modeled by agents that follow a daily plan of activities and trips. The simulation iterates between two major components: In the mobility simulation (called mobsim in Fig. 1), demand is simulated on the physical network while every agent executes its selected plan. Traffic flow is modeled by spatial first-in-first-out queues that represent links. A vehicle is allowed to leave a queue (i.e. a link), when there is no vehicle in front of it in the queue, when the link's free flow travel time has passed, when the flow capacity of the link is not exceeded in the given time step, and when there is space on the next link. Because of congestion, travel times and activity durations of the executed activity travel pattern may differ from the plan. The second mayor component of the iterative process is the mental simulation: Agents evaluate their decisions (called scoring in Fig. 1) and possibly replan them (called replanning in Fig. 1). Plans are evaluated based on their performance, which is quantified by a score. Agents are allowed to select a plan for the next iteration. A certain percentage of agents is chosen to generate a new plan by modifying an existing plan. Possible modification strategies are e.g. route, time, or mode choice. The remaining agents select one of their existing plans through probabilistic selection by a multinomial logit model, where the selection probability of a plan is related to its score. The iterative process is repeated until agent scores do not vary anymore. If scores converge, the process leads to a (stochastic) user equilibrium (SUE), i.e. no user may improve their score by unilaterally changing their strategy.

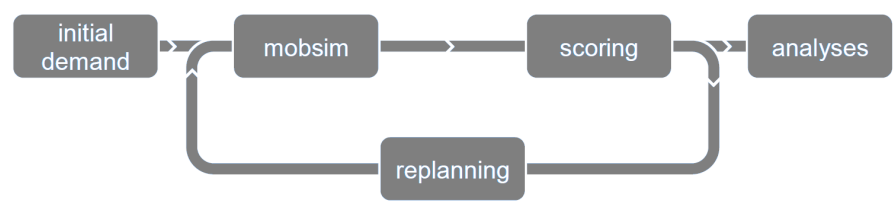

Fig. 1. Iterative cycle of the multi-agent transport simulation MATSim [6].

\section{B. Signal control in MATSim}

Traffic signals and lanes are modeled in MATSim by an extension module [7]. If a signal exists on a link, leaving the link is not possible while it shows red. The signal control unit in MATSim is called every second to possibly update signal settings. With this, traffic-responsive signals can be easily modeled.

1) Fixed-time signals: First studies in MATSim focused on fixed-time signals [8]. They strictly follow a predefined plan. The plan defines cycle times for each intersection (called signal systems in MATSim) and contains the information when to switch green and red for all incoming links of the signal system. Green waves can be modeled in MATSim via the offset values per signal system, which define the time when the signal system starts with second zero from the plan.

2) Traffic-actuated signal control SYLVIA: Traffic-actuated signals adapt an underlying fixed-time plan depending on sensor data. The control considered in this paper shortens each green stage of the fixed-time plan to $5 \mathrm{~s}$ and only extends it if vehicles are approaching. A maximal extension of 1.5 times the green time of the fixed-time plan is used. The control was implemented in MATSim by Grether, Bischoff and Nagel [9] and is based on the approach SYLVIA by Schlothauer \& Wauer [10]. Despite its simple algorithm, this signal control significantly improves travel times compared to fixed-time signals.

3) Traffic-adaptive signal control by Lämmer: In contrast to traffic-actuated signals, traffic-adaptive signals do not rely on a fixed-time plan and decide about signal settings on the fly. The algorithm considered here is based on an a priori assignment of signals into disjoint signal groups. It selects the next signal group to show green only based on local sensor data of approaching vehicles. Thereby, it minimizes waiting times while also ensuring stability. The algorithm was developed by Lämmer and Helbing [11] and implemented in MATSim and extended to cope with more realistic traffic situations in previous studies [12], [13].

\section{$C$. The combined process of signal and user reaction in MATSim}

With fixed-time signals, users adapt over the iterations to find their best (or a good) route based on the fixed signal settings and decisions of other users. This reaction takes place in the replanning part of MATSim depicted in Fig. 1. With traffic-responsive signals, signal settings depend on user decisions and, therefore, change when users change their routes. Thereby, the signal reaction is part of the mobsim module in MATSim. With this, the combined process of user reaction (from iteration to iteration based on scores of executed plans and travel times in the network) and signal reaction (from second to second based on positions of vehicles on the network) is part of the standard iterative cycle and automatically modeled in MATSim.

\section{REAL-WORLD APPLICATION}

\section{A. The scenario}

The scenario considered in this study depicts the City of Cottbus, Germany, and its surrounding county. 22 signalized intersections in the inner city are modeled (depicted in Fig. 2). 
Base case fixed-time signal plans are taken from Strehler [14]. These fixed-time plans are also used to derive input data for the responsive signals (as described in previous studies [13]). Transport demand consists of approx. 33300 commuters traveling from home to work in the morning and back to home in the evening. It is constructed based on commuter statistics. Locations for home and work activities (depicted in Fig. 2) are generated according to land use information. (See Grether [8] for a detailed description of the scenario.)

The base case is iterated until a stable state is found (SUE). Thereby, $10 \%$ of the agents choose new routes each iteration in the replanning step (see Fig. 1). These routes are than taken as input for all policy cases (i.e. other signal approaches). This means that in the first iteration signals can be compared based on same routes for all agents, whereas the last iteration represents the signal control performance under user reaction, here route choice. See Fig. 3 for an illustration.

\section{B. Effects of user adaption on the results}

Fig. 4 shows aggregated results for travel times, traveled distance and delays (i.e. waiting times due to congestion and signals) for the first iteration (corresponding to the case without user adaption) and the last iteration (i.e. including re-routed traffic). Traffic-responsive signals improve overall results compared to fixed-time signals - with and without user adoption. With user adoption, more direct routes are taken and traveled distances decrease by around $11,000 \mathrm{~km}$ (in average $170 \mathrm{~m}$ per trip) for both responsive signal approaches. Delays increase compared to the case without route choice, because more travelers use potentially delayed routes (by signals or congestion) trough the inner city. Compared to fixed-time signals, responsive signals still result in lower overall delays, though. An interesting result is, that despite increasing delays, total travel time for responsive signals further reduces when rerouted traffic is considered. Compared to fixed-time signals, total travel time is reduced by approx. $650 \mathrm{~h}\left(2.3 \times 10^{6} \mathrm{~s}\right)$ which corresponds to a travel time gain of approx. 0.2 mio $\mathrm{h} / \mathrm{yr}$. With this, the implementation of traffic-responsive signals in Cottbus is comparable to other transport policies currently discussed for that area, as e.g. a collection of by-pass routes ${ }^{1}$ with a predicted travel time gain of $0.3 \mathrm{mio} \mathrm{h} / \mathrm{yr}$. (With an installation cost of approx. 35 mio EUR, the discussed bypass routes are a lot more expensive as the installation of responsive signals including sensors at 22 intersections would probably cost with approx. 3-9 mio EUR.)

Compatible to the reduced total distance traveled, Fig. 5 verifies that traffic is induced to the inner-city when route choice is enabled: Approx. 1500 more trips use routes through the inner city which increases the total distance traveled in the inner-city subnetwork (highlighted in red in Fig. 2) by approx. $4,000 \mathrm{~km}$. This is due to significantly decreased travel times and delays in the inner city by the new signal control (see left bars in Fig. 5). Considering route choice, almost all of this reduction in travel time and delay in the inner city has

\footnotetext{
${ }^{1}$ https://www.bvwp-projekte.de/strasse/B169-G30-BB/B169-G30-BB.html
}

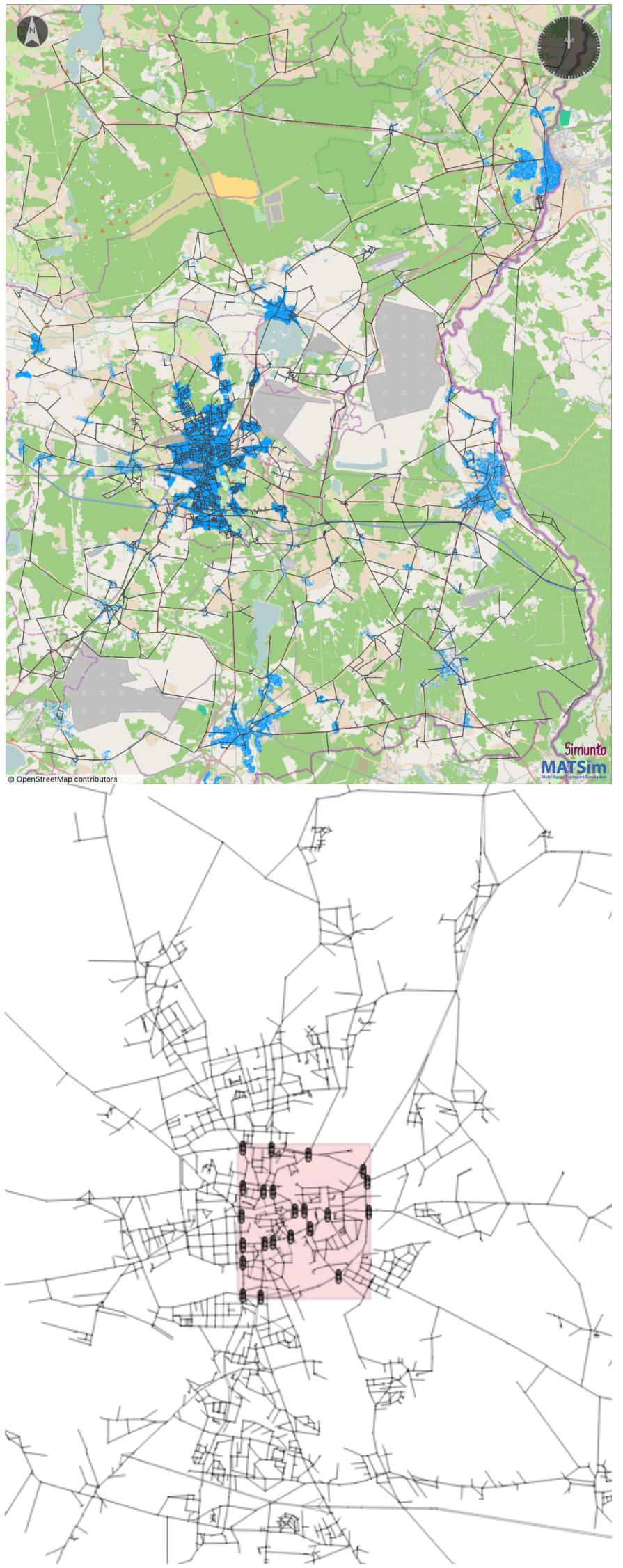

Fig. 2. Scenario of the city of Cottbus. Above: Full network with home activities in blue. Below: Inner city area highlighted in pink with signalized intersections. 


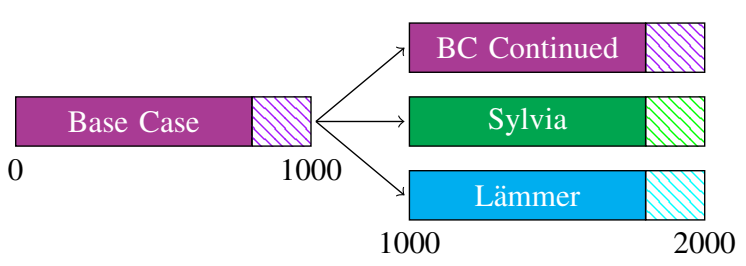

Iterations

Fig. 3. Schematic illustration of base and policy cases. 1000 iterations each (innovative strategies, i.e. route choice, is switched off after 800 iterations).
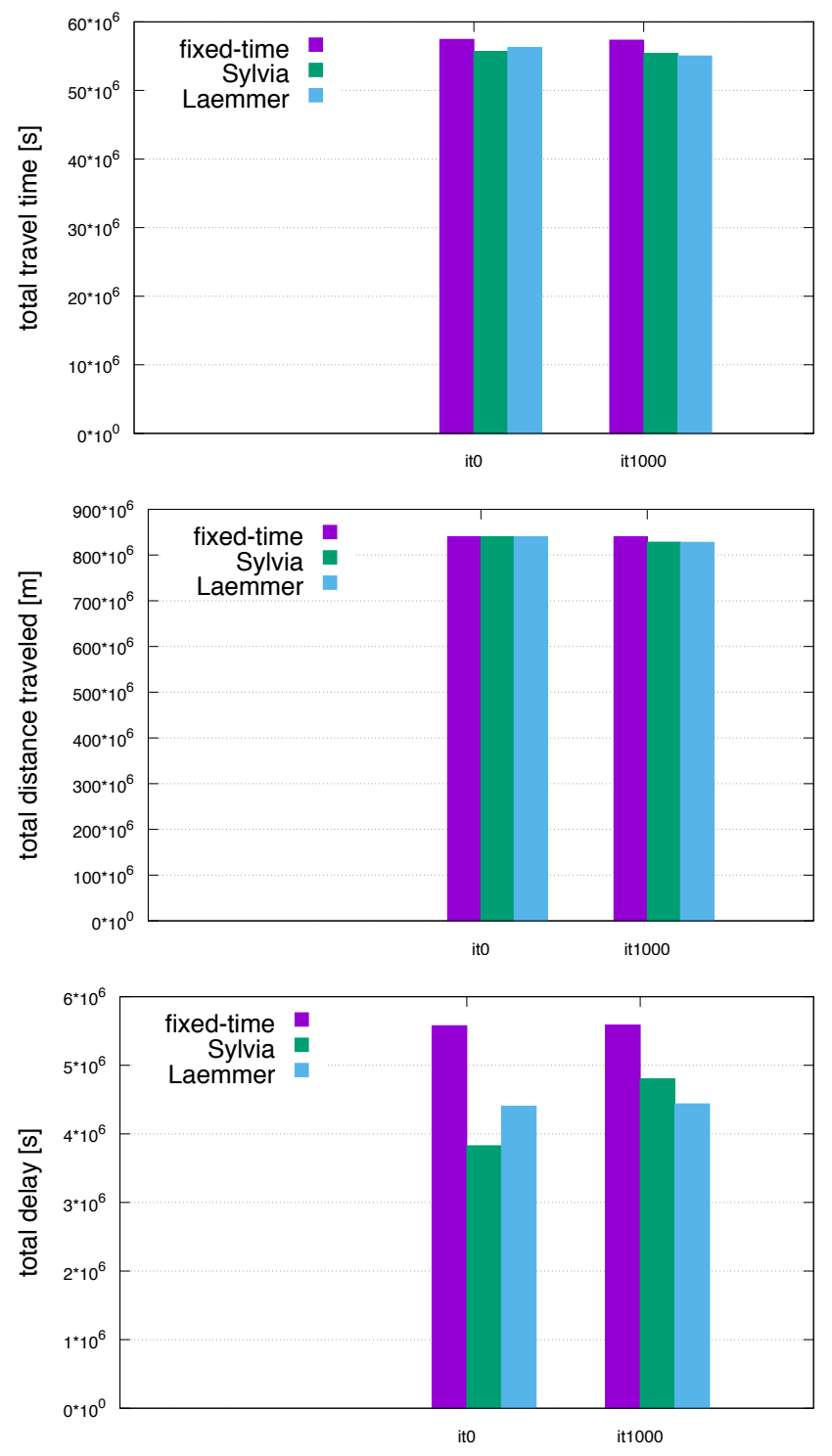

Fig. 4. Total travel time, traveled distance and total delay in the full network without vs. with route coice. vanished, though. For the Lämmer control a travel time gain of $104 \mathrm{~h}$ in the inner city (compared to fixed-time signals) is left; for Sylvia only $10 \mathrm{~h}$. On the other hand, this also means that despite the significantly increased number of travelers through the inner city, travel times and delays in the inner city do not increase - but even slightly decrease.

Spatial re-routing effects can be observed in the upper part of Fig. 6 which shows a difference plot of traffic volumes between fixed-time signals and the adaptive signals by Lämmer. It can be seen that more traffic uses inner city streets, by-pass routes become less congested (green colored routes in the figure) and major routes leading into the inner city become more congested (colored red). The lower part of Fig. 6 illustrates the difference in average delays on the links of the network between fixed-time and Lämmer signals. Despite the reduced overall delay due to more flexible signal control, average delay on most inner city intersections increases (colored in red) because of the higher number of travelers there.

With shorter distances traveled and more congestion, namely in the inner city, people working or living in the inner city do not necessarily benefit from the new signal control. This can also be verified when analyzing the emissions and noise level in the inner city. Fig. 7 shows results of a noise calculation in MATSim [15]. As expected from the traffic volumes, the noise level in the inner city and on the major roads is the highest (see upper part of Fig. 7 which shows the absolute noise immissions with fixed-time signals). With traffic-responsive signals, noise immissions in the inner city are even higher, because of the induced traffic (see lower part of Fig. 7 which shows the difference in noise immissions between fixed-time and Lämmer signals). Some noise reduction can be observed on the city by-pass routes (colored yellow and green in the figure). These effects would be even higher, when damages would be considered (i.e. weighted by the number of people who experience the noise immissions) because more people live and work in the inner-city area. The effect that people in the inner city do not necessarily benefit from the new signal control, would not be visible when re-routing traffic was not analyzed.

\section{Effects of different levels of saturation on re-routed traffic}

Effects of user reaction and induced traffic might be different for different levels of saturation. The results presented in the previous section were based on the fixed level of saturation that the scenario of the city of Cottbus has in it's base version. However, in MATSim one can easily scale link capacities up or down to simulate a lower or higher level of saturation. Therefore, saturation flow values (i.e. link flow capacities) and storage capacity values of the links are multiplied by a flow capacity factor. With this, one can represent situations with less or more congestion without the need to change the demand such that traffic volumes and number of trips etc. stay comparable between the different levels of saturation.

This section analyzes the effects of the so up- and downscaled scenario of the city of Cottbus regarding the influence of user adoption. Fig. 8 shows travel times, traveled distances 

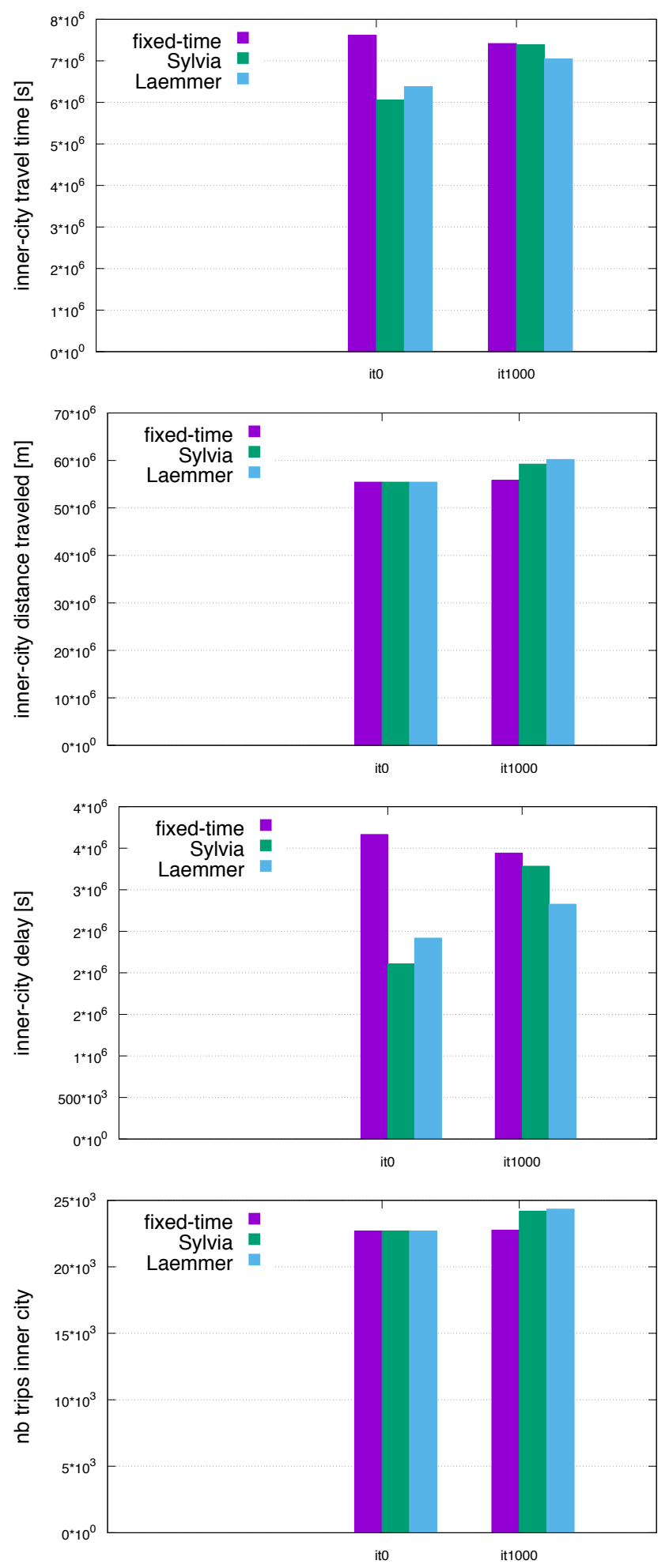

Fig. 5. Total travel time, traveled distance, total delay and number of trips in/through the inner-city subnetwork (highlighted in red in Fig. 2).

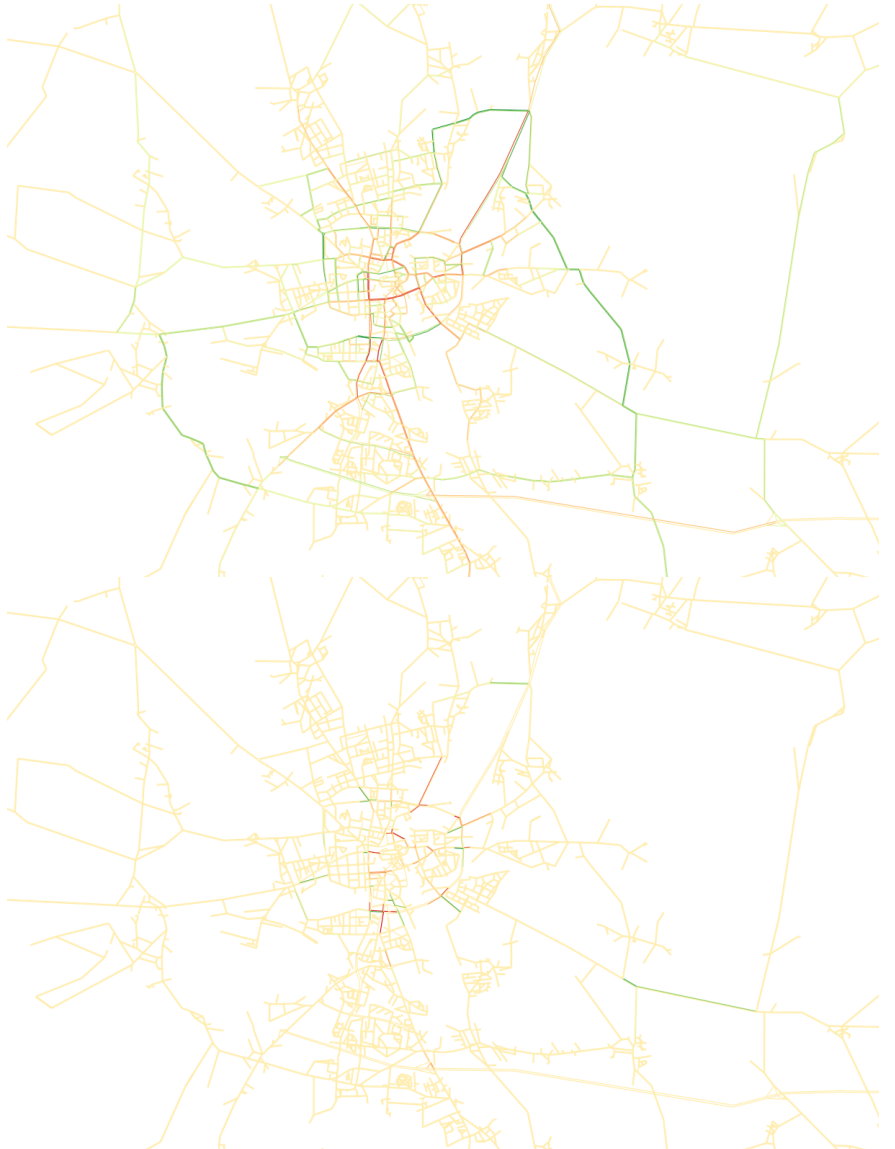

Fig. 6. Differences in total traffic volumes (above) and average delays per vehicle per link (below) of fixed-time vs. Lämmer signals. (Red means traffic volumes, or delays, respectively, have increased with Lämmer signals; green means the decreased.)

and number of trips through the inner city for different levels of saturation. Note, that the base version of the scenario (used in the previous section) corresponds to a flow capacity factor of 0.7 because this factor produced most realistic values for travel time and congestion levels compared to real-world traffic counts, see [8].

The number of trips through the inner city (see lower part of Fig. 8) shows, that the effect of induced traffic is higher the more flexibility the system has, i.e. the lower the level of saturation. For lower flow capacity values (i.e. higher saturation), the number of induced trips is lower. Driving through the congested inner city gets less attractive with increasing level of saturation - no matter which signal control is used. Travelers prefer wider by-pass routes which are less affected by congestion when travel time through the inner city increases (see the middle and lower plot in Fig. 8). Overall, both responsive signal algorithms produce similar results for different levels of saturation. Induced traffic effects are most similar for flow capacity factors between 0.5 and 0.7 . For higher flow capacity values (i.e. less saturation), Lämmer signals induce slightly more traffic through the inner city than Sylvia signals, due to more flexibility that the algorithm has 

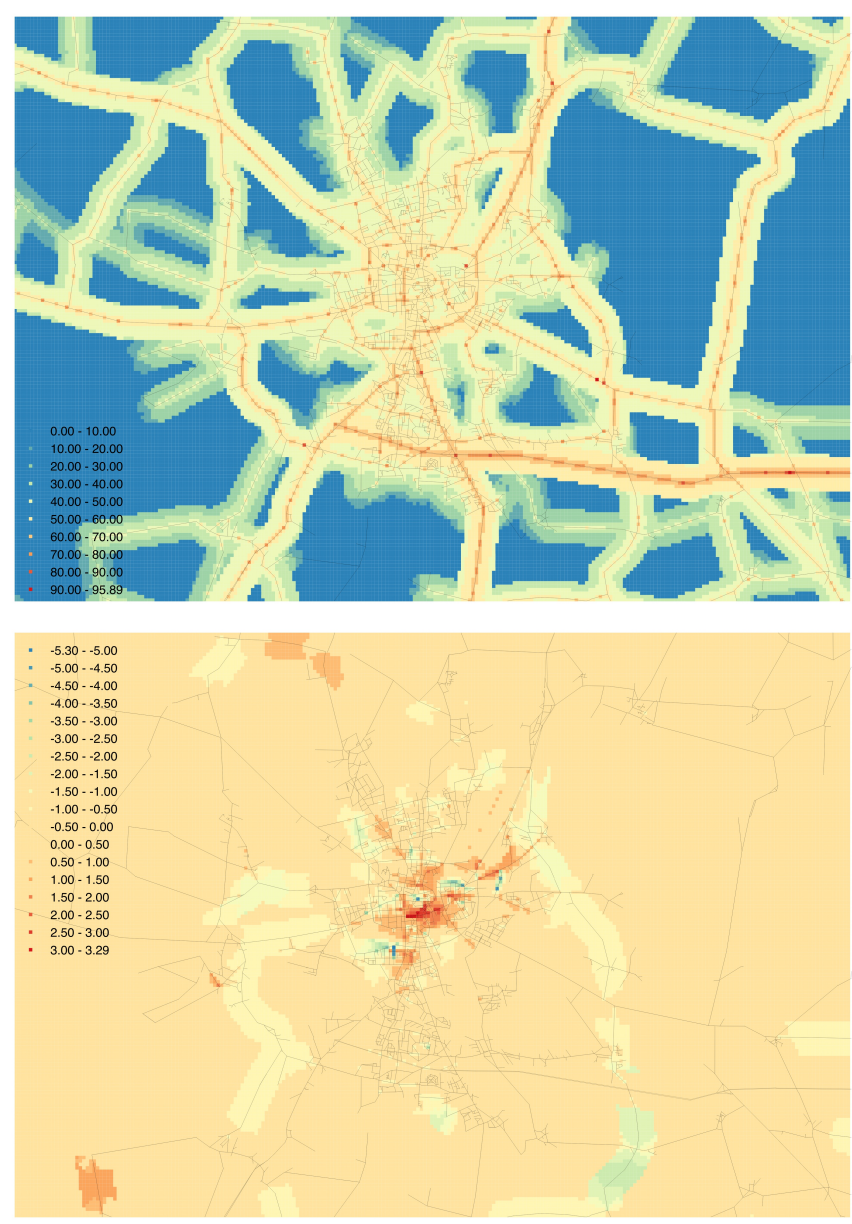

Fig. 7. Absolute noise immissions with fixed-time signals in the morning peak (above) and differences in noise immissions with fixed-time vs. Lämmer signals (below). (Red means higher immissions with Lämmer signals; yellow and green means lower immissions.)

especially for uncongested situations. Contrary to the effect that the lower plot of Fig. 8 suggests, improvements in travel time and traveled distance by responsive signals increase with increasing levels of congestion: Absolute improvements in traveled distance increase from approx. $7000 \mathrm{~km}$ to $1300 \mathrm{~km}$; absolute travel time gains significantly increase from $430 \mathrm{~h}$ to $1300 \mathrm{~h}$ (from the flow capacity factor of 0.9 to the flow capacity factor of 0.4).

\section{CONCLUSION}

This study shows that induced traffic matters when modeling traffic signal control. When evaluating control approaches it is not enough to analyze travel times and delays for the current demand. User reaction (e.g. route choice) significantly influences the results: Traveled distances decrease because travelers choose more direct routes through the inner city, traffic volumes and, therefore, noise levels in the inner city increase and also travel time and delay compared to the case without user reaction increases. I.e. people living or working in the inner city do not necessarily benefit from the new signal
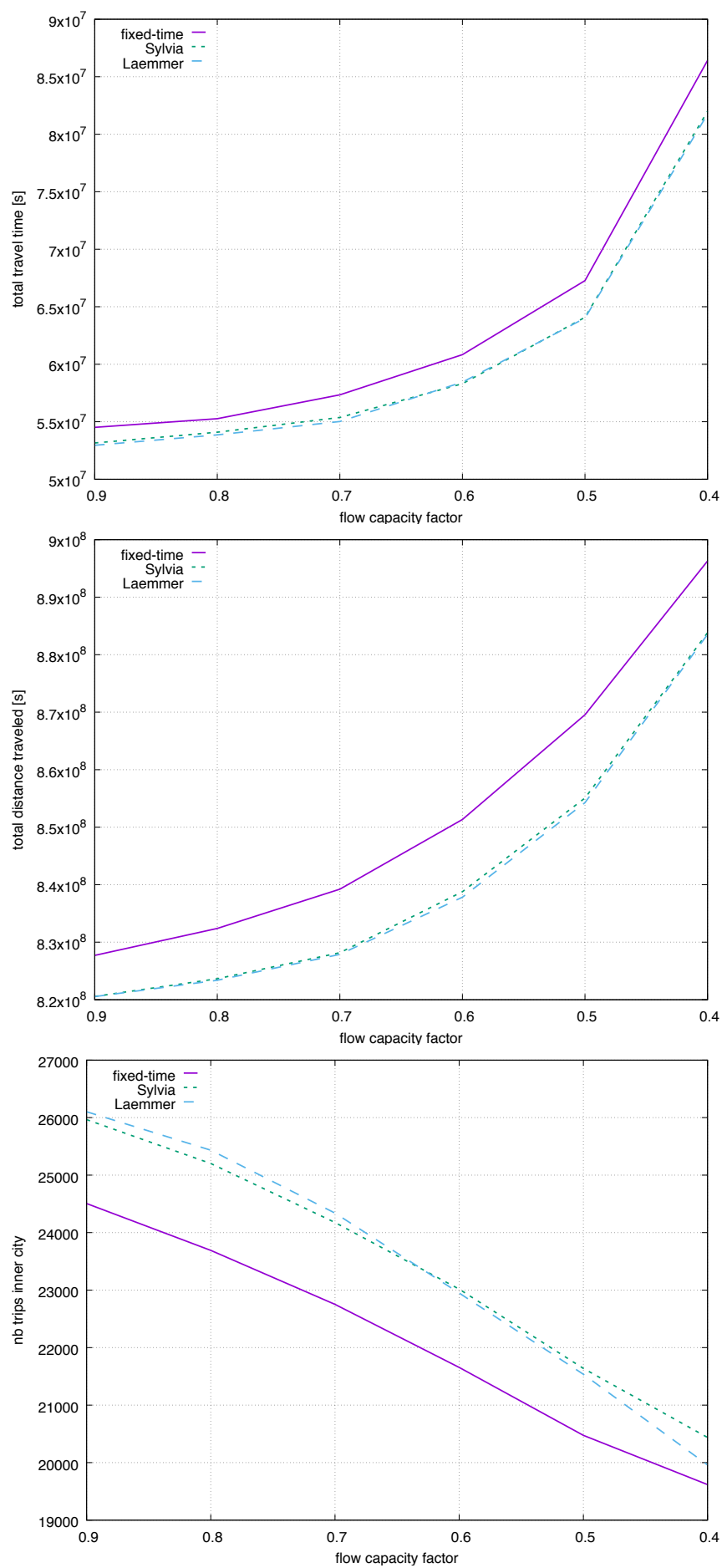

Fig. 8. Travel time, traveled distance and number of trips through the inner city for different levels of saturation (i.e. different flow capacity factors). 
control. Nevertheless, a significantly improvement in travel time and delay is observed with traffic-responsive signals compared to fixed-time signals in this study, even when rerouting effects are modeled. Furthermore, it is found that the level of saturation has an impact on the effects of user adoption on the results: The higher the overall saturation, the lower the number of induced trips through the inner city, but, on the other hand, the higher the potential improvement in travel time and traveled distance by traffic-responsive signals.

Other user reactions than route choice, such as mode or time choice, potentially enable even stronger effects on induced traffic, because of their higher flexibility. An interesting next step, therefore, is to repeat the experiments from this study with mode and/or time choice. Also, it would be interesting to analyze, whether results of this study are affected when agent are allowed to adapt simultaneously to traffic-signals, i.e. from second to second in the simulation (which can be done in MATSim by enabling within day replanning).

\section{REFERENCES}

[1] E. Köhler and M. Strehler, "Traffic signal optimization using cyclically expanded networks," Networks, vol. 65, no. 3, pp. 244-261, 2015.

[2] T. Thunig, R. Scheffler, M. Strehler, and K. Nagel, "Optimization and simulation of fixed-time traffic signal control in real-world applications," Procedia Computer Science, in press.

[3] C. Osorio and M. Bierlaire, "A simulation-based optimization framework for urban transportation problems," Operations Research, vol. 61, no. 6, pp. 1333-1345, 2013.

[4] M. Smith and T. van Vuren, "Traffic equilibrium with responsive traffic control," Transportation Science, vol. 27, no. 2, pp. 118-132, 1993.

[5] H. van Zuylen and H. Taale, "Urban networks with ring roads: A twolevel, three player game," Paper 04-1659, Transportation Research Board Annual Meeting, Washington, D.C., 2004.

[6] A. Horni, K. Nagel, and K. W. Axhausen, eds., The Multi-Agent Transport Simulation MATSim. Ubiquity, London, 2016.

[7] D. Grether and T. Thunig, "Traffic signals and lanes," in Horni et al. [6], ch. 12.

[8] D. S. Grether, Extension of a Multi-Agent Transport Simulation for Traffic Signal Control and Air Transport Systems. PhD thesis, TU Berlin, Berlin, 2014.

[9] D. Grether, J. Bischoff, and K. Nagel, "Traffic-actuated signal control: Simulation of the user benefits in a big event real-world scenario," in $2 n d$ International Conference on Models and Technologies for ITS, Leuven, Belgium, 2011. Also VSP WP 11-12, see http://www.vsp.tu-berlin.de/ publications.

[10] Schlothauer \& Wauer Ingenieurgesellschaft für Straßenverkehr $\mathrm{mbH} \&$ Co. KG, "SYLVIA+ short description." https://www.schlothauer.de/en/software-systems/sylvia/, 2011. Website version: $30-07-2018$.

[11] S. Lämmer and D. Helbing, "Self-control of traffic lights and vehicle flows in urban road networks," Journal of Statistical Mechanics: Theory and Experiment, vol. 2008, no. 04, pp. 04-019, 2008.

[12] N. Kühnel, T. Thunig, and K. Nagel, "Implementing an adaptive traffic signal control algorithm in an agent-based transport simulation," Procedia Computer Science, vol. 130, pp. 894-899, 2018.

[13] T. Thunig, N. Kühnel, and K. Nagel, "Adaptive traffic signal control for real-world scenarios in agent-based transport simulations," Transportation Research Procedia, forthcoming.

[14] M. Strehler, Signalized flows - optimizing traffic signals and guideposts and related network flow problems. PhD thesis, BTU Cottbus, 2012.

[15] I. Kaddoura, L. Kröger, and K. Nagel, "An activity-based and dynamic approach to calculate road traffic noise damages," Transportation Research Part D: Transport and Environment, vol. 54, pp. 335-347, 2017. 